\title{
Possibilities and Limitations of Photoactivatable Cytochalasin D for the Spatiotemporal Regulation of Actin Dynamics
}

Roshna V. Nair, ${ }^{[a]}$ Shifang Zhao, ${ }^{[a, b]}$ Emmanuel Terriac, ${ }^{[a]}$ Franziska Lautenschläger, ${ }^{[a, c]}$ Joseph H.R. Hetmanski, ${ }^{[\mathrm{d}]}$ Patrick T. Caswell, ${ }^{[\mathrm{d}]}$ Aránzazu del Campo*a,b]

(a) INM - Leibniz Institute for New Materials. Campus D2 2, 66123 Saarbrücken. Germany

(b) Saarland University, Chemistry Department, 66123 Saarbrücken, Germany

(c) Saarland University, Physics Department, 66123 Saarbrücken, Germany

(d) Wellcome Trust Center for Cell-Matrix Research. Faculty of Biology. University of Manchtester. Manchester Academic Health Science Centre, Manchester, UK

*corresponding author: aranzazu.delcampo@leibniz-inm.de

\begin{abstract}
The study of the actin cytoskeleton and related cellular processes requires tools to specifically interfere with actin dynamics in living cell cultures, ideally with spatiotemporal control and compatible with real time imaging. A phototriggerable derivative of the actin disruptor Cytochalasin D (CytoD) is described and tested here. It includes a nitroveratryloxycarbonyl (Nvoc) photoremovable protecting group (PPG) at the hydroxyl group at C7 of CytoD. The attachment of the PPG renders Nvoc-CytoD temporarily inactive, and enables light-dosed delivery of the active drug CytoD to living cells. This article presents the full structural and physicochemical characterization, the toxicity analysis. It is complemented with biological tests to show the time scales (seconds) and spatial resolution (cellular level) achievable with a UV source in a regular microscopy setup.
\end{abstract}

\section{Introduction}

The cellular cytoskeleton is a dynamic network with relevant functions in cell force generation and transmission. Being able to regulate the dynamics of specific cytoskeletal elements enables targeted studies of force-related cellular processes. ${ }^{1-2}$ An established strategy to control cytoskeletal dynamics is by adding small molecule inhibitors to the cell culture that selectively interact with individual cytoskeletal components. In order to regulate the activity of a drug in a living cell with spatiotemporal resolution, photoactivatable and photoswitchable drug derivatives have been developed ${ }^{3}$. In the first case, the drug is modified with a photoremovable protecting group (PPG) at a relevant position for binding to its target. ${ }^{4-6}$ This chemical modification significantly reduces the affinity of the drug for the cytoskeletal component, inhibiting its bioactivity. The latent activity of the photoactivatable drug allows the drug to be in the cellular milieu without causing any biological effect. Light exposure at a given time point cleaves the PPG and activates function at the exposed site and at its surroundings, at a time scale that depends on diffusion. Two examples of photoactivatable drugs to control the dynamics of microtubules have been reported. A photoactivatable derivative of CMPD1, a tubulin polymerization inhibitor, was obtained by attaching the photoremovable protecting group 4,5-dimethoxy-2-nitrobenzyl (DMNB). ${ }^{7}$ A similar strategy was used to locally deliver paclitaxel (PTX), a drug that inhibits depolymerization of microtubules. ${ }^{6,8}$ Variants with DMNB and 6-bromo-7-hydroxycoumarin-4-yl derivatives have been reported, among others. In contrast to photoactivatable drugs, photoswitchable drugs are rely on modifications with azobenzene-based photoswitches, and allow reversible variation between a latent and an active state by exposure to light of different wavelenghts. As 
example, photoswitchable azobenzene photostatins to photocontrol microtubule dynamics have been reported and tested in vivo. ${ }^{9}$

Among drugs affecting the actin network, the fungal metabolites Cytochalasins ${ }^{10-11}$ are potent candidates. They are disruptors of the actin network organization by barbed-end capping mechanism, and the inhibition of G- and F-actin binding to cofilin. ${ }^{12-13}$ When added as drug to cell cultures, cells experience decreased stiffness, ${ }^{14}$ ruffling of the membrane,${ }^{10,15}$ enucleation, ${ }^{16}$ or arborization. ${ }^{17}$ Although Cytochalasins interact with actin in several ways, which complicates the analysis of cellular responses, ${ }^{12-13}$ they are widely used in cell biology and biophysics to externally interfere with the actin network and investigate cytoskeleton dynamics and related cellular processes. From the 20 naturally occurring types of cytochalasins, cytochalasin D (CytoD) shows the highest specificity for actin. ${ }^{18}$ CytoD shows no binding to glucose transporter, and no inhibition of monosaccharide transport across the plasma membrane, unlike cytochalasins $\mathrm{A}$ and $\mathrm{B} .{ }^{19} \mathrm{CytoD}$ shows a 10 times higher binding affinity for F-actin than the other cytochalasin types. ${ }^{18}$ Its potent actin polymerization inhibitory nature is advantageous for local studies of actin dynamics, in spite of having a multimodal actin-disruption mechanism, but creates the necessity for fine control of CytoD dose.

In this article, the synthesis and detailed characterization of physicochemical properties, photochemical activity and toxicity of a phototriggerable derivative of CytoD are described. This compound enables light-dosed delivery of CytoD in living cells, and was used in previous works to regulate actin dynamics in cell biology studies. ${ }^{20,21} \mathrm{We}$ analyze the time scale and spatial resolution at which actin control is realizable in living cells. This compound facilitates studies in cell biology by allowing regulation of a relevant element in the cellular force generation and transmission machinery at higher precision than a soluble drug.

\section{Results and Discussion}

\section{Design and synthesis of Nvoc-CytoD}

The crystal structure of CytoD bound to monomeric actin has been reported. ${ }^{22}$ The graphical representation of actin-CytoD interaction site (Figure 1) shows that the isoindolone core (comprising the lactam ring bearing benzyl group and the $-\mathrm{OH}$ at $\mathrm{C} 7$ ) interacts with actin via five hydrogen bonds. The fused large 11-membered macrocycle containing a tertiary $-\mathrm{OH}$ exhibits only one H-bonding interaction. The positioning of CytoD is also strongly guided by hydrophobic interactions at the back half of the hydrophobic cleft between subdomains 1 and 3. The relevant residues of actin for the stabilization of CytoD in the binding pocket are Ile136, Tyr169, Ala170, Pro172, Met355, and Phe375. Taking into account the chemical structure and reactivity of CytoD, and the interaction sites with monomeric actin, the secondary hydroxyl group at $\mathrm{C} 7(-\mathrm{OH})$ seems to be the most adequate position for caging (Figure S1, S2). C7 hydroxyl group interacts through water-mediated hydrogen bonds with carbonyl of V134 and NH of I136. Introduction of a PPG at this position would presumably affect the two $\mathrm{H}$ bonds and destabilize the complex. For this study the PPG 3,4-dimethoxy nitroverytryloxocarbonyl (Nvoc) was selected. Nvoc-CytoD was synthesized in one step by reacting CytoD with Nvoc-Cl (Figure $\mathrm{S} 1$ ). The reaction occurred selectively, only at the secondary $\mathrm{C} 7$ hydroxyl, with no interference of the less reactive tertiary hydroxyl at $\mathrm{C} 18$. Nvoc-CytoD was obtained in $68 \%$ yield after purification. The high specificity and reasonable yield of this reaction is important, since CytoD is a very expensive drug available at $\mathrm{mg}$ scale. The chemical structure of the product was confirmed by 1H-NMR and mass spectrometry. $\mathrm{H} 7$ 
signal shifted from $\delta 3.83 \mathrm{ppm}$ in CytoD (1H NMR in CDCl3) to $\delta 5.00-4.96 \mathrm{ppm}(1 \mathrm{H}, \mathrm{d}, \mathrm{J}=$ $11 \mathrm{~Hz}$ ) in Nvoc-CytoD as consequence of deshielding by the carbamate group. A downfield shift of H8 signal was also observed, from $2.67 \mathrm{ppm}$ in CytoD to 3.22-3.15 ppm in NvocCytoD (Figure S2a). Nvoc-CytoD was readily soluble in DMSO, chloroform and dichloromethane. Nvoc-CytoD was stable in PBS ( $\mathrm{pH}=7.4)$ and 4\% DMSO for at least 24 hours. HPLC analysis of the solution showed no hydrolysis during this time (Figure S3). However, insolubilization and formation of aggregates was noticed within a few hours.

\section{Photochemical properties}

The photolysis of Nvoc-CytoD was followed by UV spectrophotometry and HPLC studies of solutions irradiated at $\lambda_{\max }=360 \mathrm{~nm}$ for increasing times. The UV spectra of Nvoc-CytoD showed an absorption maximum at $343 \mathrm{~nm}$, as consequence of the presence of the Nvoc (Figure 2B). Upon exposure, a drop of absorbance at $\lambda_{\max }$, was observed, in parallel to a rise of absorption intensity around $400 \mathrm{~nm}$. Similar changes in the UV profile have been reported for other Nvoc-photolysis cases and are attributed to the formation of the nitroso byproduct during photolysis. ${ }^{8,23-24}$ In the HPLC curves, the intensity of the Nvoc-CytoD peak (retention time $16.6 \mathrm{~min}$ ) decreased, while a new peak of increasing intensity appeared at retention time of $13 \mathrm{~min}$ (Figure 2A). Mass analysis confirmed that this peak corresponded to free CytoD. At full exposure at $360 \mathrm{~nm}, 70 \%$ of Nvoc-CytoD was photolyzed and $64 \%$ of CytoD was formed (Figure 2C), according to HPLC quantification. Longer exposure doses did not improve this ratio and lead to formation of other photodegradation byproducts, which we were not able to identify (Figure 2A).

\section{Activity of the drug and toxicity tests}

The cytotoxicity of Nvoc-CytoD was assessed first in a live/dead assay. This experiment was done on confluent monolayers of MDCK cells incubated overnight with either CytoD or Nvoc-CytoD in the concentration range of $10 \mathrm{nM}$ to $50 \mu \mathrm{M}$. Cell viability ratios dropped to $70 \%$ at $0.1 \mu \mathrm{M}$ CytoD incubation concentration. In contrast, viability $>90 \%$ were observed for Nvoc-CytoD at concentrations up to $1 \mu \mathrm{M}$. These results demonstrate a significant lower toxicity of CytoD by introducing the Nvoc PPG at C7 in its molecular structure. Viability dropped to $80 \%$ at $5 \mu \mathrm{M}$ Nvoc-CytoD incubation concentration, which represent the experimental condition selected for the photoactivation experiments. Although this ratio of cell death is not negligible, it is important to note that the conditions used to evaluate cytotoxicity (overnight incubation) are never applied in a cell biology study. Shorter incubation times are expected to be much more benign to the cells. The observed cytotoxicity of Nvoc-CytoD at $>5 \mu \mathrm{M}$ could be due to enzymatic cleavage of the carbamate group and consequent release of CytoD.

We further examined the bioactivity of Nvoc-CytoD by measuring changes in the spreading area of cells after incubation with the drug, since cell spreading is very sensitive to changes in the actin fibers. For this experiment epithelial MDCK cells were not convenient since these cells form clusters and collective effects could counteract the effect of the drug. Mesenchymal-like hTert-RPE1 cells were selected, as these cells can be kept isolated in culture when using low cell seeding density. The quantification of the cell area after 7 hours incubation with CytoD and Nvoc-CytoD is shown in Figure 2B. A decrease in cell area was clearly visible in cells incubated with CytoD at concentrations $>0.02 \mu \mathrm{M}$, and cells were found to detach from the culture plate at concentrations $>10 \mu \mathrm{M}$. In contrast, cells incubated with Nvoc-CytoD did not show visible changes in spreading area at concentrations $<1 \mu \mathrm{M}$. 
These results confirm that Nvoc-CytoD affects the survival rate and the shape of cells at significant lower extent than CytoD.

The effect of Nvoc-CytoD treatment on the actin cytoskeleton was visualized in live imaging experiments at shorter incubation times, i.e. 1-3 hours, as used in later cell experiments. For this purpose L929 fibroblasts were first labelled with Sir-actin by overnight incubation. ${ }^{25}$ Upon addition of $0.1 \mu \mathrm{M}$ CytoD the F-actin was immediately disrupted and cells were not able to spread properly. Figure S4 shows exemplary images taken after 3 hours incubation with the drug. L929 cells exposed to Nvoc-CytoD did not show visible signs of a disrupted cytoskeleton at any incubation concentration up to $50 \mu \mathrm{M}$. These results further confirm the drastic change in CytoD activity upon introduction of the Nvoc group, and reflect the concentration range at which Nvoc-CytoD can be used for experimentation.

\section{Demonstration of light-induced changes in actin using Nvoc-CytoD in cell cultures}

The experimental conditions to use Nvoc-CytoD as a photoactivatable drug to interfere with the actin cytoskeleton of living cells upon light exposure were tested in different experiments using MDCK, RPE1, L929 and MEF cell types. Actin was labelled with SiRactin (for MDCK, L929 and MEFs) or with LifeAct (for RPE1 and A2780). Experiments were designed to identify the minimum incubation concentration necessary to induce local actin changes without appreciable photodamage to the cells, and to explore the resolution in time and space and the degree of control at which actin can be disrupted or regulated using Nvoc-CytoD. In preliminary experiments, MEFs labelled with SiR-actin were exposed to $50 \mu \mathrm{M}$ NvocCytoD and a defined intracellular area (ca. $180 \mu \mathrm{m}^{2}$ ) containing visible actin stress fibers was irradiated (Figure 4A). Neither visible changes in the spreading area nor in the organization of the actin cytoskeleton were visible in the presence of the non active drug, as observed in previous experiments with L929 (Figure S4). Upon illumination, we observed a fast fluorescence loss within the irradiated area, both in Nvoc-CytoD treated and control samples, indicating bleaching of the F-actin fluorophore at the exposure conditions. Fluorescence recovered after $10 \mathrm{~min}$ in the control experiment and showed cells with similar F-actin structures as before exposure, demonstrating that the irradiation step alone was not affecting the actin structures. In contrast, cells treated with Nvoc-CytoD and illuminated showed a distorted actin cytoskeleton with clusters of actin and actin-free areas after fluorescence recovery (Figure 4A). The observed features in the F-actin cytoskeleton are expected for a treatment with CytoD. These results confirm that Nvoc-CytoD can diffuse inside the cells and liberate active CytoD when irradiated. They also highlight that bleaching of the fluorophore precludes fluorescence imaging of actin disruption during the recovery time of the fluorophore. In these experiments the strongly associated reporter SiR-actin was used, which has the exchange kinetics in the order of minutes. This agrees with the observed 10 minutes necessary for full recovery of the initial fluorescence pattern. Eventually a weaker associated F-actin reporter (eg. LifeAct with exchange kinetics in the order of seconds) could shorten this time lag.

In order to avoid bleaching of the actin label and to minimize cell photodamage during intracellular exposure step, we performed a similar experiment by irradiating a region outside of the cell, close to the cell membrane. We hypothesized that the free CytoD would diffuse from the illuminated region into the cytosol of the cells. In fact, Figure 4B shows the actin cytoskeleton $10 \mathrm{~min}$ after irradiation of a $180 \mu \mathrm{m} 2$ area outside the MEF cell. A disrupted actin fibers and formation of actin clusters was observed. Also cellular structures dependent on the actin network, like focal adhesions ${ }^{26}$, were affected. The total area and average size of the focal adhesions decreased after exposure (Figure 4C,D). In contrast, no significant 
changes in the actin organization or the focal adhesions were observed in the control experiments. These results demonstrate the possibility to deliver CytoD, to avoid bleaching of actin and to image early actin changes by irradiating extracellular space close to the imaging site. It is important to note that the drug concentration at the imaging site will depend on the Nvoc-CytoD concentration, the exposure dose, and also on the diffusion rate of the molecules from the exposed site to the imaging site. Diffusion will occur in a gradient form and cannot be easily quantified. This is a limitation of this approach. A time lapse experiment using RPE1 cells expressing LifeAct shows that the diffusion of activated CytoD and subsequent actin disruption occur already within the first minute after exposure (video S1, Figure S5). The changes in the actin cytoskeleton as a consequence of light activation of Nvoc-CytoD were temporal. In many cases (not all) cells recovered an organized actin cytoskeleton within less than one hour (MEFs in Figure S6) within a time frame spanning from 5 min to 1 hour depending on the concentration of CytoD liberated in the experiment, and eventually the exposure conditions and cell type. This is a consequence of the dilution of the locally liberated drug in the medium and allows successive experiments within a cell culture without changing the medium. This cannot be achieved upon incubation with $\mathrm{CytoD}$, since all cells are exposed to the same drug concentration at a time.

We then explored the spatial resolution at which delivery of CytoD is possible by local activation of NVoc-CytoD at subcellular level. For this purpose a small region close to the leading edge of migrating A2780 cells was exposed. Local delivery next to small protrusions reduced the intensity of Lifeact-mEmerald and led to collapse of the protrusion within 5 minutes, with recovery of actin polymerization at later times (Figure 5A). When Nvoc-CytoD was activated in a small region close to larger lamellipodial protrusions, the local effect of CytoD was clearly observed by the splitting of lamellipodia due to disassembly of F-actin close to the irradiated region (Figure 5C,D), whereas by irradiation in the absence of NvocCytoD lamellipodia remained intact (Figure 5B). The snap shots from 20 minute time lapse (Figure 5D) show that the protrusion close to photorelease site loses F-actin (loss of LifeActEmerald signal) up until around 10 mins, at which point F-actin identity begins to increase again, presumably after the effective concentration of photoreleased Nvoc-CytoD decreases due to diffusion. These data demonstrate that Nvoc-CytoD can be released in a highly targeted manner. Reducing the area of irradiation and time of activation can lead to local disruption of actin networks at a subcellular level in cells without the cellular damage associated with conventional photoablation methods.

In a different experimental setting, we explored the limits of the use of Nvoc-CytoD to interfere with the actin cytoskeleton of a cell embedded in a confluent monolayer of epithelial cells $^{20}$. It is important to note that, compared to the widely used laser ablation method, ${ }^{27}$ Nvoc-CytoD is specific towards the cytosolic component that is destabilized. In this experiment we sought to destabilize the equilibrium of traction forces within the monolayer by illuminating a $6 \times 7 \mu \mathrm{m}^{2}$ cytosolic area of a single MDCK cell within the monolayer treated with Nvoc-CytoD. Dissociation of the actin stress fibers in the illuminated cell was observed already 1 minute after exposure (SiR-actin labelling, Figure 6A,B). The changes started at the illuminated area. They propagated through the cell at $45 \mathrm{~nm} / \mathrm{s}$ during the first 160 seconds, and at lower rate $(31 \mathrm{~nm} / \mathrm{s})$ for 3 minutes afterwards (Figure 6C). At 5 minutes the actin cytoskeleton of neighbouring cells also appeared distorted (Figure 6A, * symbol), while the cytoskeleton of the second neighbours was less affected (Figure 6A, arrow). In the control irradiation experiment with no Nvoc-CytoD we observed bleaching of the actin staining after illumination, followed by fluorescence recovery within $50 \mathrm{~s}$ time scale (Figure S6), and no changes in the actin structure. Note that the destabilization of the actin cytoskeleton fibers in the monolayer is a consequence of two parallel effects: the CytoD 
liberated upon exposure and possibly diffusing to neighboring cells, and the natural cytoskeletal rearrangements upon loss of the force equilibrium inside the monolayer. The differentiation between the two contributions is not possible in our experiment, and is a potential experimental limitation of Nvoc-CytoD and disadvantage vs. laser ablation method. We hypothesize that optimization of the experimental conditions by minimizing the photoliberated CytoD concentration and the irradiated volume could allow reducing the effect of drug diffusion to the single cell level.

The local concentration of photoreleased CytoD in the cell culture cannot be easily determined. This is a limitation of this method. However, the relative concentration and the drug release profile can be varied by simply changing illumination parameters. In order to visualize this possibility, we monitored the change in the cell area of a MDCK cell in a monolayer after different exposure conditions. The distortion of the actin structure upon illumination is expected to create a temporal force imbalance that could result in a cell shape change. To quantify cell area we imaged E-cadherin signal at the median plane of the cell. Exposure of a MDCK cell of the monolayer to a defined exposure dose ( 5 illumination pulses at $\mathrm{t}=0 \mathrm{~min}$ ) resulted in an abrupt increase of cell area within the first minute, followed by a slower increase up to a final $10 \%$ increase of the initial cell area during 5 minutes (Figure 7A and B). This result is in agreement with an expected impaired ability of the cell to apply cytoskeletal contraction forces. The cell area remained constant for longer time points, i.e. at 10 min (Figure 7C) or 30 min (data not shown). In contrast, the area of a MDCK cell exposed at 1 pulse per minute during 5 minutes showed a continuous increase of the cell area and reached the same $10 \%$ increase at $5 \mathrm{~min}$. When the exposure dose was doubled (5 more pulses per minute in the following 5 minutes), a net $20 \%$ increase in cell area was observed. This experiment visualizes how exposure profiles can be utilized to regulate CytoD concentration in time and space.

Altogether, these results show that Nvoc-CytoD allows localized delivery of CytoD at cellular level in cell culture and offers a reasonable degree of control on the delivered drug amount and pharmacokinetic profile. All experiments were conducted with low intensity illumination at $405 \mathrm{~nm}$. Control experiments verified that the used exposure doses did not cause any changes in the actin organization by themselves. These results highlight the potential and limitations of Nvoc-CytoD to locally disturb the filamentous actin within a cell. This compound has proven useful in the study of dilution of cortex induced cell super elasticity behavior in epithelial domes..$^{20}$ Operating with Nvoc-CytoD is simple, as it can easily added to the cell culture medium. This is an advantage vs. other existing approaches for actin regulation relying on microinjected photoactivatable actin-binding proteins (photoactivatable Thymosin $\beta 4$ applied to photocontrol actin dynamics and the direction of migration of keratocytes $^{28}$ or photoactivatable Cofilin tested with MTLn3 cells ${ }^{29}$ ) or on optogenetic engineering of the cell of interest. ${ }^{30-31}$

Nvoc-CytoD opens the door to interesting experiments in cellular biology and mechanotransduction. A few examples are: i) the study of mechanical tension within cell monolayers (i.e. epithelium) and the response cell-cell junctions to actin alterations; ${ }^{20}$ ii) the function of local actin dynamics in control of cell structure and organization (e.g. during cell division, cell migration); ${ }^{21}$ iii) localised cross talk between actin organization and cell-cell and cell-matrix adhesion; iv) roles of actin within the cell nucleus.

\section{Conclusions}


Nvoc-CytoD is a photoactivatable derivative of CytoD that can be added to the medium and allows light-mediated local delivery of the CytoD drug within a few seconds. Using NvocCytoD and controlled light exposure at the outer side of the cellular membrane, the actin network can be affected at subcellular level within time scales of tens of seconds to minutes. The pharmacokinetic profile of the free drug can be controlled by light exposure, but diffusion causes the free drug to be diluted in the medium in a gradient-form, making quantification of liberated CytoD complicated. Diffusion allows local CytoD concentration to be diluted and actin distortion to be reverted depending on the drug concentration. These features allow multiple experiments with the same cell, or in the same dish without washing steps or medium change. The experiments can be performed using a normal confocal microscope equipped with a $405 \mathrm{~nm}$ laser (without photo-manipulation unit). Activation of Nvoc-CytoD by intracellular irradiation is also possible, though the required light dose leads to extensive bleaching of labelled actin and hinders imaging of actin dynamics during recovery time (ca. 10 mins). Advanced PPGs with higher photosensitivity could overcome this problem and push the limits of temporal and spatial resolution and accurate quantification in advanced biological questions. Experiments in this direction are in progress. 


\section{Experimental Section}

\section{Cell culture and sample preparation}

Mouse Fibroblasts L929 (NCTC clone, ATCC) were cultured in RPMI 1640 medium (GIBCO) supplemented with 10\% fetal calf serum (FCS; GIBCO). WT eGFP-Vinculin mouse embryonic fibroblast lines (MEFs) were cultured in DMEM with $10 \%$ fetal bovine serum, $1 \%$ Nonessential Amino Acids, 1\% Sodium Pyruvate and $4 \mathrm{mM}$ L-Glutamine (all of them purchase from GIBCO). ${ }^{32}$ L929 and MEFs expressing Vinculin-GFP were cultured in a humidified incubator at $37 \mathrm{C} / 5 \% \mathrm{CO} 2$. Madin-Darby Canine Kidney (MDCK) cells stably expressing E-Cadherin-RFP were cultured in DMEM medium (Gibco) with 10\% fetal bovine serum (Fischer scientific), 1\% GlutaMAX (Fischer Scientific) and 1\% penycilin/streptomycin (Gibco).

SiR-actin was used for actin imaging. After cell attachment, cell culture medium was replaced by fresh medium containing SiR-actin $(100 \mathrm{nM})$. After $12 \mathrm{~h}$ incubation F-actin was labeled by Sir-actin. Then Nvoc-CytoD in DMSO was added to the medium to a final concentration of $50 \mu \mathrm{M}$ Nvoc-cytoD or $0.1 \%$ DMSO. DMSO was used for the control group.

Retinal Pigment Epithelial cells (h-tert RPE1) were genetically modified by infection in order to stably express LifeAct-mcherry, a filamentous actin reporter. ${ }^{33}$ Cells were cultured in DMEM/F-12 medium supplemented with $10 \%$ fetal bovine serum, $1 \%$ penicillin/streptomycin and $1 \%$ Glutamax (all of them purchased from GIBCO) in a humidified incubator at $37^{\circ} \mathrm{c}$ and $5 \% \mathrm{CO} 2$.

For sample preparation for microscopy, $0.5 \mathrm{ml}$ of cell suspension $(30.000$ cells $/ \mathrm{ml})$ was filled into each of the 4 compartments of cell culture dish CELLview ${ }^{\mathrm{TM}}$.

\section{Live-Cell Imaging}

Time-lapse recordings were acquired with 20x, 63x objectives using either a Cell Observer inverted microscope (Zeiss Axio Observer Z1) controlled by ZEN blue software, a confocal microscope (Zeiss LSM 880) controlled by ZEN black software or an inverted Nikon TiEclipse microscope equipped with a Yokogawa CSU-W1 spinning disk head and a FRAPPA module (both from Andor Technology) for photoactivation. All microscopes were equipped with an incubation chamber which maintained the temperature at $37{ }^{\circ} \mathrm{C}$ and $\mathrm{CO} 2$ concentration at $5 \%$.

\section{In situ photolysis of Nvoc-CytoD}

A selected position in the cell culture was scanned using a Zeiss LSM 880 with a $405 \mathrm{~nm}$ laser (laser power $0.234 \mathrm{~mW}$ ) for 45 seconds. Briefly, the selected position was zoomed in full field of view, then took a picture by $405 \mathrm{~nm}$ laser $(108.7 \mu$ s per pixel, image area is 179.99 $\mu \mathrm{m} 2$ ). The irradiation area was labeled with square by ZEN black software. For inside cell irradiation, $5-15 \%$ of maximal intensity of the laser laser (nominal power of the laser: $30 \mathrm{~mW}$ ) was used through a 60x Apochromat objective (Zeiss), while 100\% laser power was used for outside cell irradiation. Images of the cells were recorded shortly before and $10 \mathrm{~min}$ after irradiation. For time lapse movies, images were acquired every 30 seconds during $10 \mathrm{~min}$ using a $63 x$ oil objective after irradiation.

\section{Toxicity assays}

Live-dead assay. MDCK cells were grown to confluence in 96 well plates for 24 hours. Cell medium was then exchanged for fresh medium containing different concentration $(0.001-$ $100 \mu \mathrm{M})$ of CytoD or Nvoc-CytoD. After one night, the cell medium was removed and exchanged by PBS containing $8 \mu \mathrm{M}$ Fluorescein diacetate (ThermoFisher scientific) (live cells) and $20 \mu \mathrm{M}$ propidium iodide (ThermoFisher scientific) for 5 minutes. The staining was then replaced by PBS and cells were imaged for green and red fluorescence. 
Cell area measurements. HTert-RPE1 stably expressing mcherry lifeAct cells were seeded at low density in 96 well plate and kept in incubator until they reached around $40 \%$ confluence. Cell medium was exchanged by fresh medium containing different concentration of CytoD or Nvoc-CytoD. After 7 hours incubation, 2 images per well were taken in brightfield and red fluorescence. The cell area was then determined by segmentation of the fluorescent signal and automatic particle detection with Fiji software. ${ }^{34}$

\section{Imaging and data Analysis}

The acquired fluorescent images and DIC time series were processed and analyzed with Fiji (distribution of ImageJ), or ZEN blue software, or homemade routines under Fiji (Macros). For the quantification of the size of focal adhesion, the images of MEFs before and $10 \mathrm{~min}$ after expose were acquired. To assess the size of focal adhesion, an automatic particle analysis (homemade macros) was performed and the number, area and total area of particle were measured to get the number, size and total area of focal adhesion.

\section{Statistical analysis}

For each condition a minimum of three independent experiments were performed. Data were expressed as mean \pm standard deviation. Box plots represent the middle $50 \%$ of data between the 1 st to the 3 th quartile (interquartile range IQR); the whiskers indicate variability outside the upper and lower quartiles. One-way ANOVA was used to determine significance between groups followed by a post-hoc Tukey contrast (GraphPad Software). For non-parametric data we performed Kruskal-Wallis analysis followed by Dunn's post-hoc multiple comparisons. All the cases a value of $\mathrm{p}<0.05$ was used for statistical significance.

\section{Acknowledgements}

Authors acknowledge Prof. Andrés Garcia (GeorgiaTech, USA) for providing MEFs, Prof. Matthieu Piel (Institut Curie Paris, France) for providing RPE1 cells, Dr. Marcelo J. Salierno (Max Planck Institute for Polymer Research) for support with the preliminary cell vitality experiments, Dr. Karin Kiefer for support with the toxicity test of cells, and Dr. Thomas Ruckelshausen for helping with quantification of focal adhesion and imaging. AdC acknowledges funding from the European Union's Horizon 2020 research and innovation Program No. 731957 (FET Mechanocontrol). AdC and FL acknowledge financial support from the Deutsche Forschung Gemeinschaft (SFB 1027). Shifang Zhao acknowledges scholarship from the China Scholarship Council (CSC). 
A

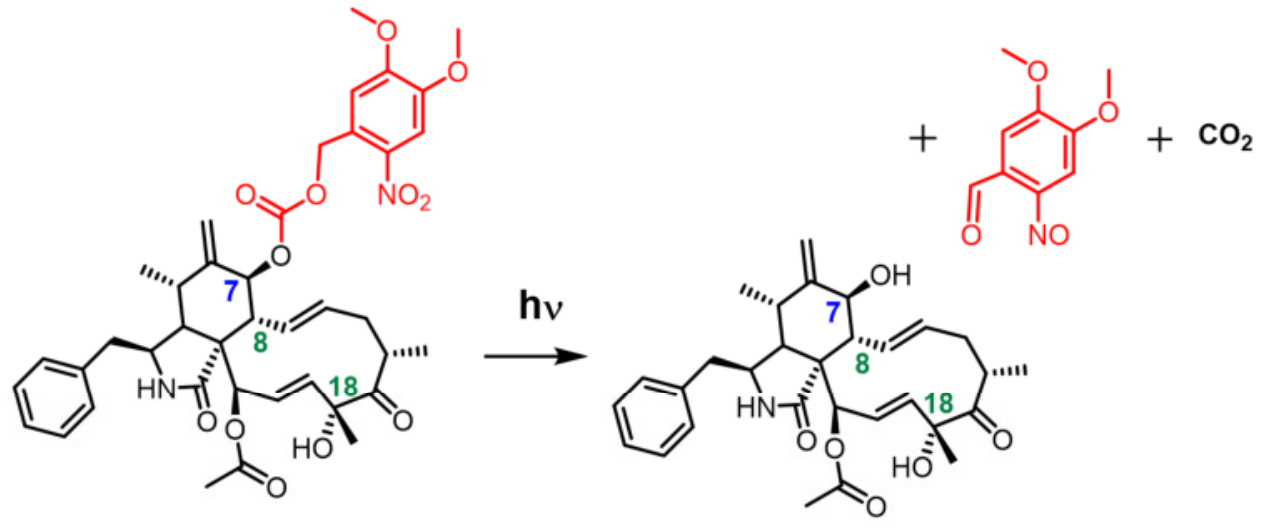

Nvoc-CytoD

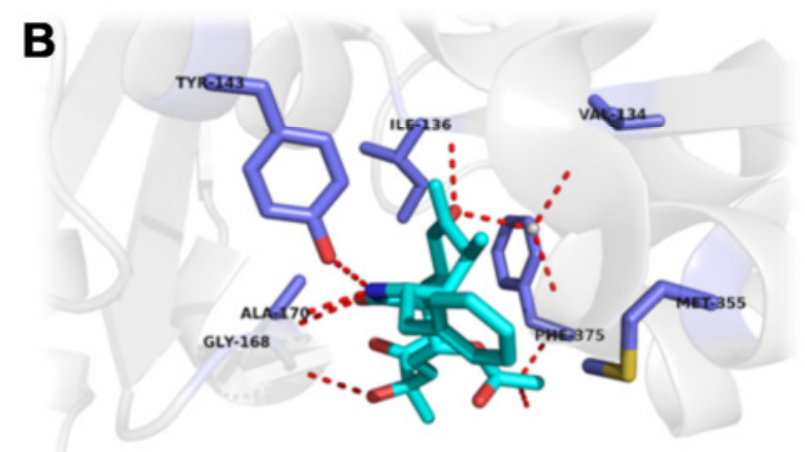

CytoD

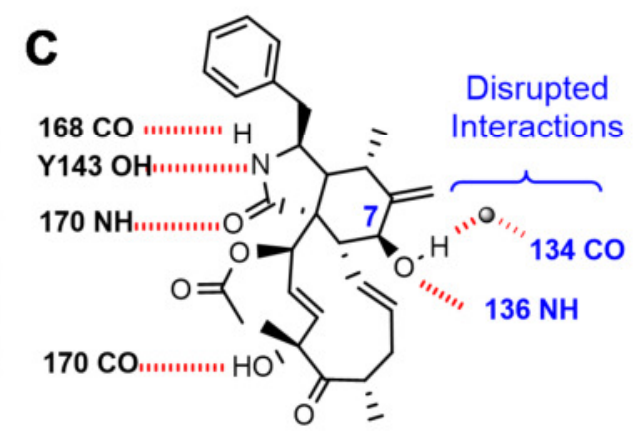

Figure 1. A) Structure of Nvoc-CytoD and its photochemical activation reaction. B) Expanded view of CytoD (cyan sticks) bound to monomer actin (grey). Hydrogen bonding interactions (red dotted lines) are highlighted. This Figure is recreated from PDB ID 3EKU, ${ }^{22}$ and rendered using PyMOL (DeLano Scientific). C) Hydrogen bonding interactions (red dotted lines) involved in CytoD-actin binding are highlighted. Note that the hydroxyl at C7 interacts with Ile136(NH) and V134(CO). 

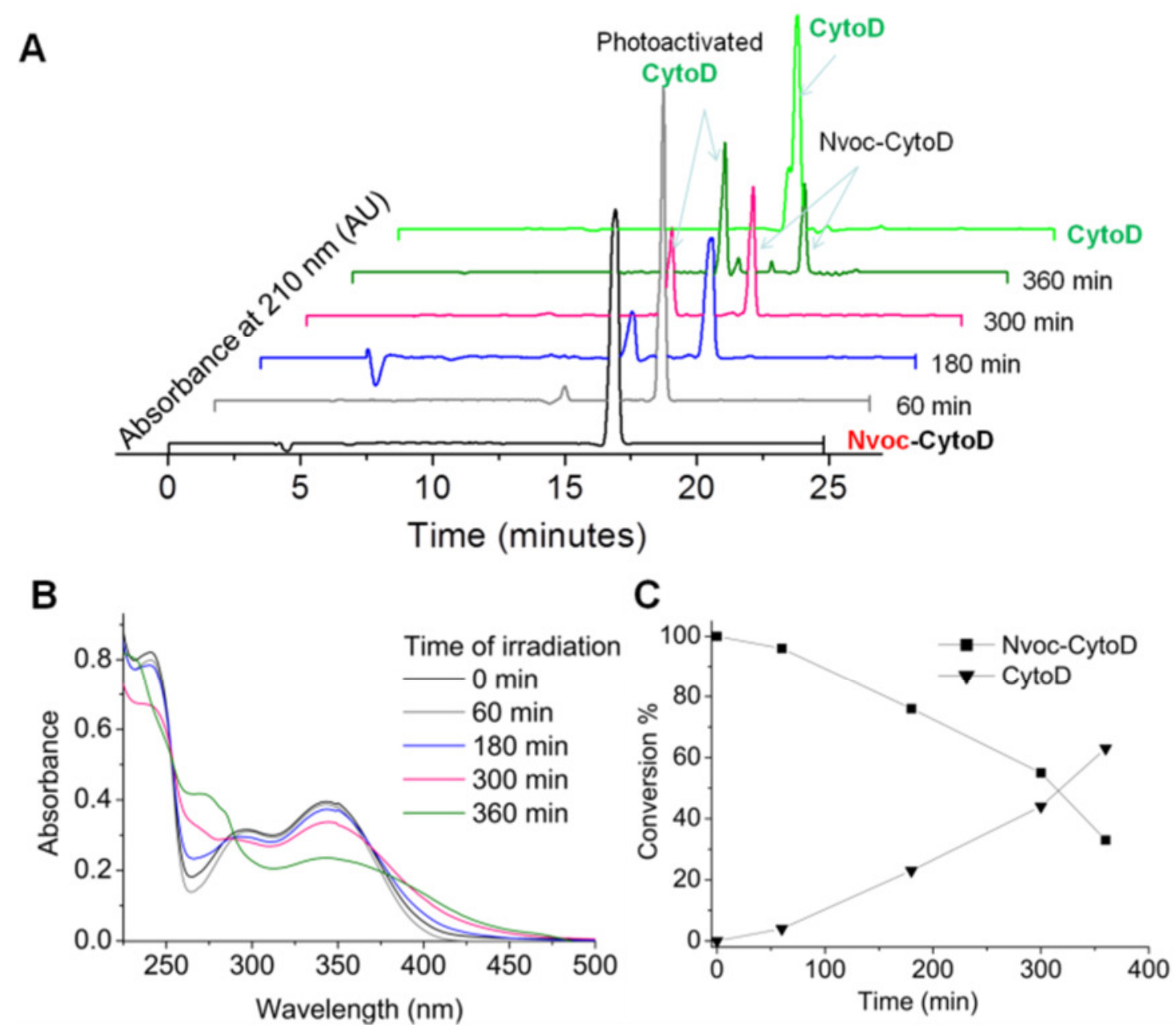

Figure 2. A) HPLC profile and B) UV-VIS spectra of a $0.55 \mathrm{mM}$ Nvoc-CytoD solution after increasing irradition time at $360 \mathrm{~nm}(2.7 \mathrm{~mW} / \mathrm{cm} 2)$. C) Quantification of the conversion degree of the photochemical reaction from HPLC analysis of irradiated solutions in A. Note that the range of drug concentration $(\mathrm{mM})$ and exposure times (hours) required for these physicochemical experiments are completely different to the conditions used in cell experiments in next Figures (local $\mu \mathrm{M}$ concentrations and seconds). 

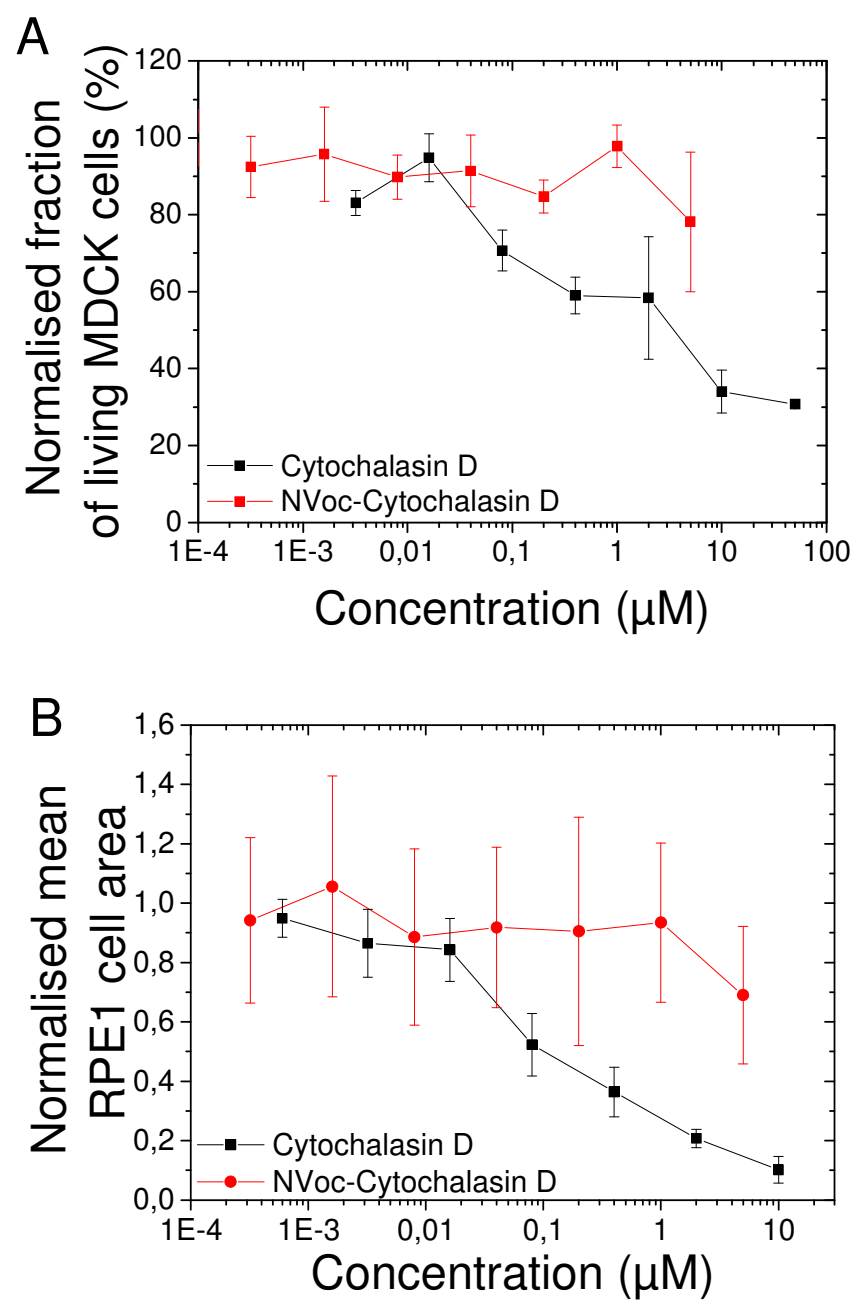

Figure 3. Toxicity and bioactivity assays A) Ratio of living MDCK cells after incubation with varying concentrations of CytoD or Nvoc-CytoD overnight. Viability ratio was calculated as percentage of viable cells compared to untreated control monolayer using live/dead assay. B) Quantification of cell area in hTert-RPE1 cultures after incubation with different concentrations of CytoD and Nvoc-CytoD for 7 hours. Cell area values were averaged for at least 50 cells per conditions (split in 2 independent experiments) and were normalized by the mean cell area for the control condition $(0.1 \%$ DMSO). Results for both experiments are represented as mean \pm standard deviation of the mean. 

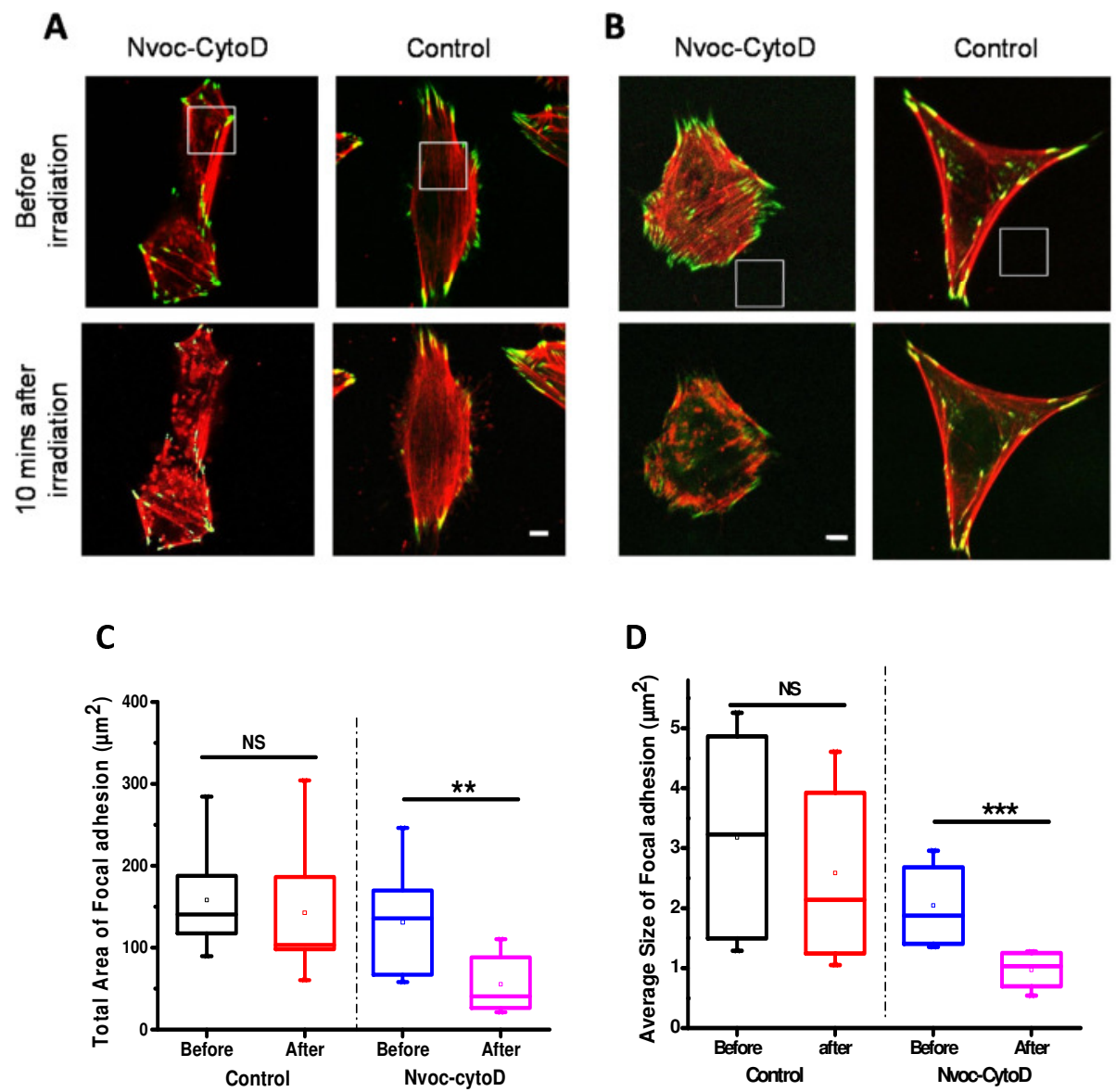

Figure 4: A,B) Representative fluorescence images of MEF cells incubated with $0.1 \%$ DMSO (control) or with $50 \mu \mathrm{M}$ Nvoc-CytoD in DMSO immediately before exposure and 10 min after exposure. Exposed region is marked with a white square and was inside the cell (A) or outside of it (B). Irradiation time was $45 \mathrm{~s}$. F-actin (red) was labelled with SiR-actin. Green represents GFP-Vinculin. Scale bar is $5 \mu \mathrm{m}$. C) The total area of Focal adhesions and, D) the average size of focal adhesion before and 10 min after light irradiation at nearby region outside the cell (as in B). ${ }^{* * *} \mathrm{p}<0.001,{ }^{* *} \mathrm{p}<0.01$, NS $=$ not significant. 


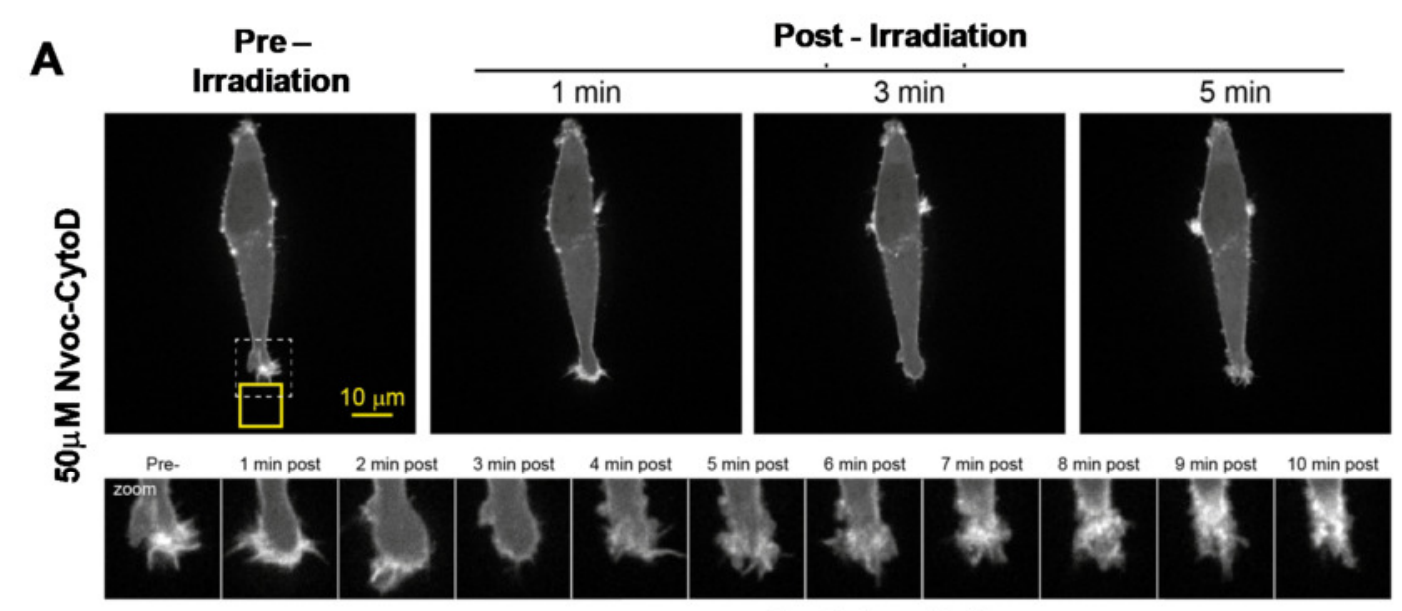

B

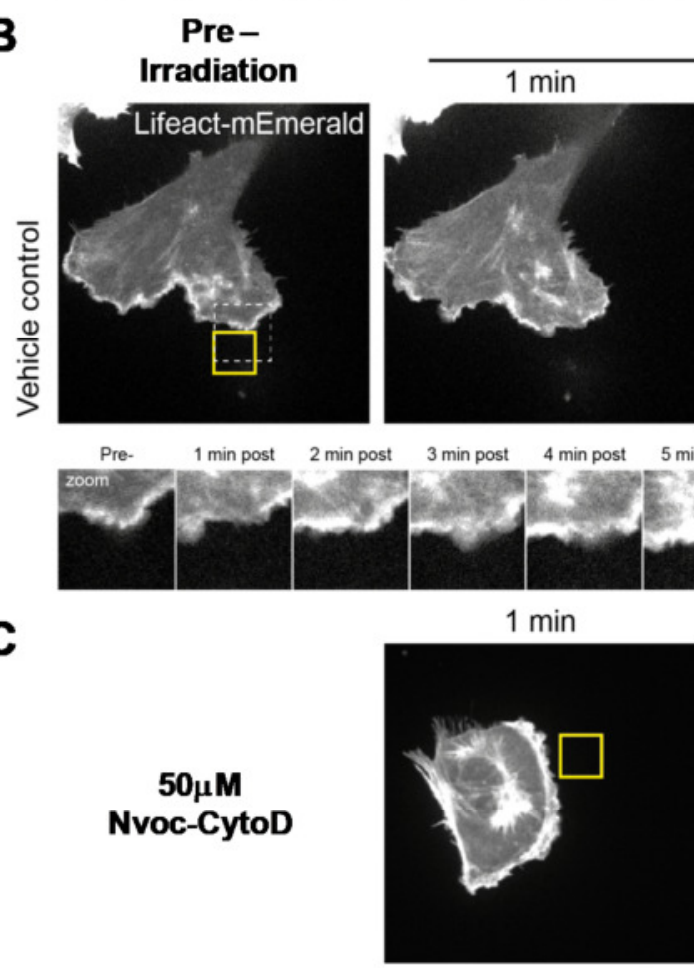

Post - Irradiation
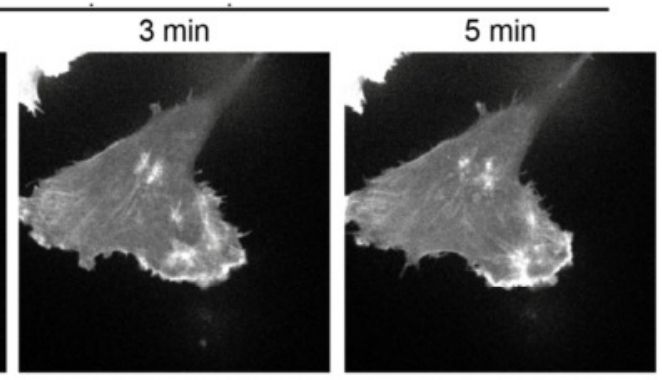

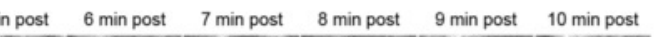
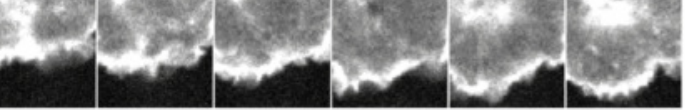

c

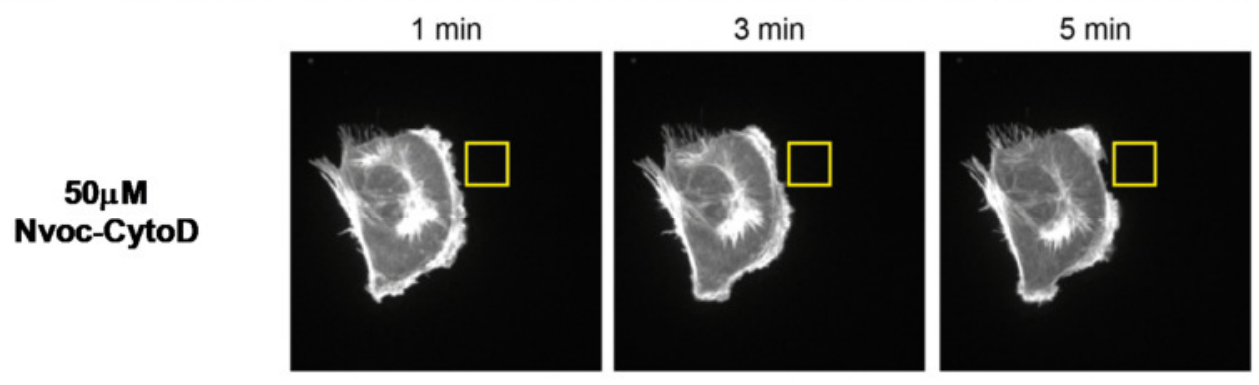

D Pre-
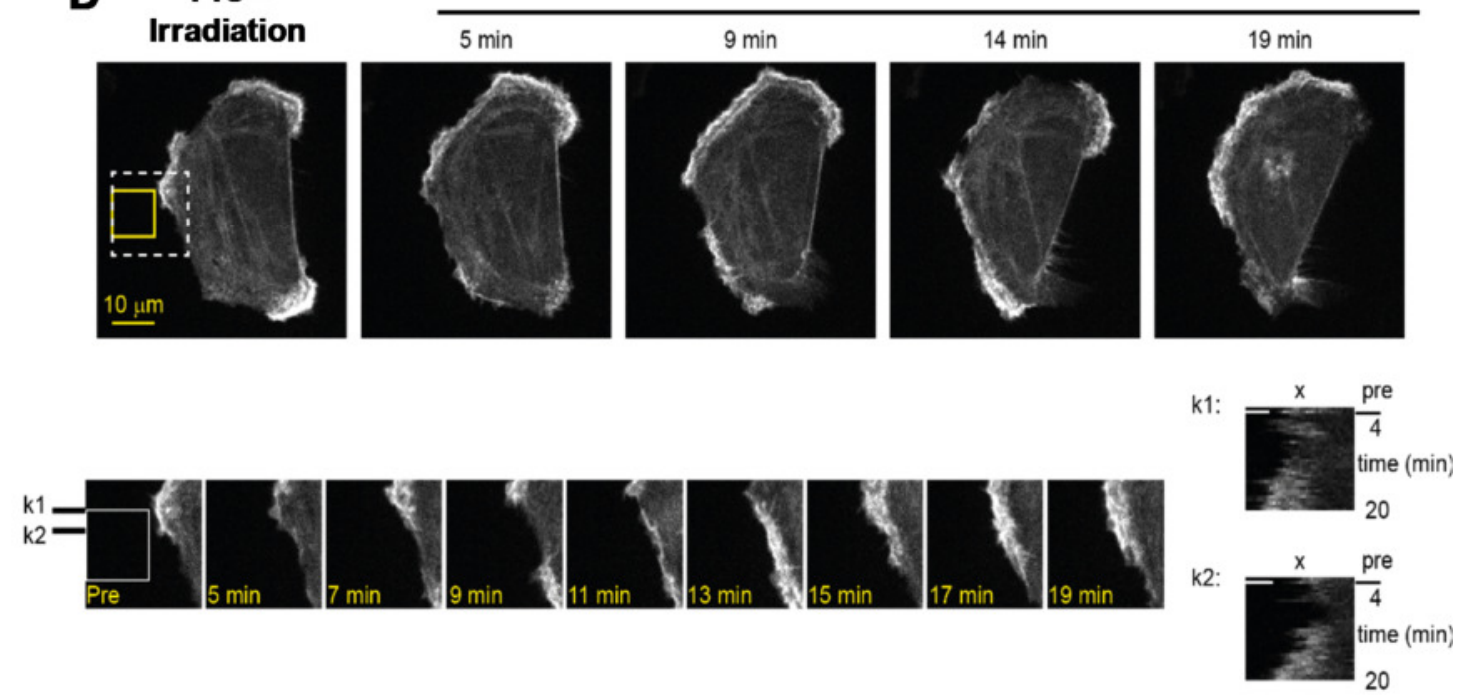
Figure 5. Photoactivatable Cytochalasin D selectively inhibits leading edge actin dynamics at subcellular resolution. A) Representative A2780 cell expressing LifeAct-mEmerald and treated with $50 \mu \mathrm{M}$ Nvoc-CytoD captured pre photo-manipulation and 1, 3 and 5 minutes post photo-manipulation (top), where the yellow box corresponds to the photo-manipulated $10 \mathrm{x}$ $10 \mu \mathrm{m} \mathrm{ROI}$, and the white dashed box corresponds to the zoomed time-course images of this leading edge region (bottom). B) Representative control A2780 cell in the presence of vehicle (i.e. the solvent and no Nvoc-CytoD) imaged as in A. C) Representative A2780 cell expressing LifeAct-mEmerald and treated with $50 \mu \mathrm{M}$ Nvoc-CytoD 1, 3 and 5 minutes postphoto-manipulation, showing clear lamellipodial disruption. D) Representative A2780 cell expressing LifeAct-mEmerald and treated with $50 \mu \mathrm{M}$ Nvoc-CytoD pre-photomanipulation and 5-19 minutes post-photo-manipulation. 
A

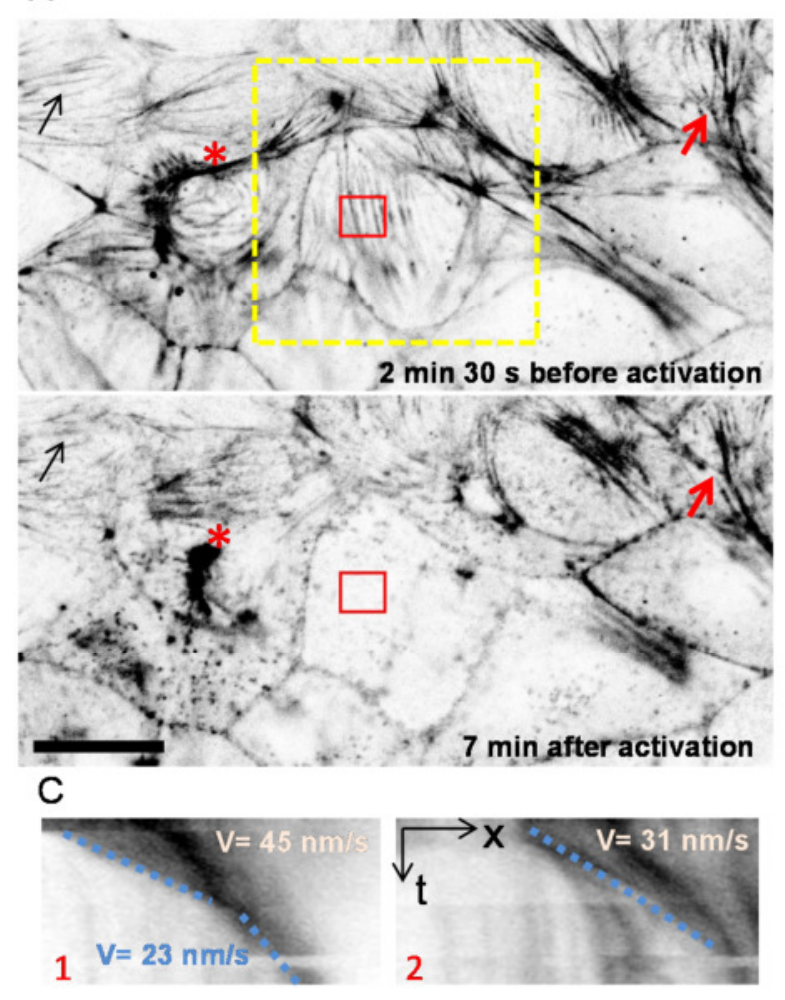

B
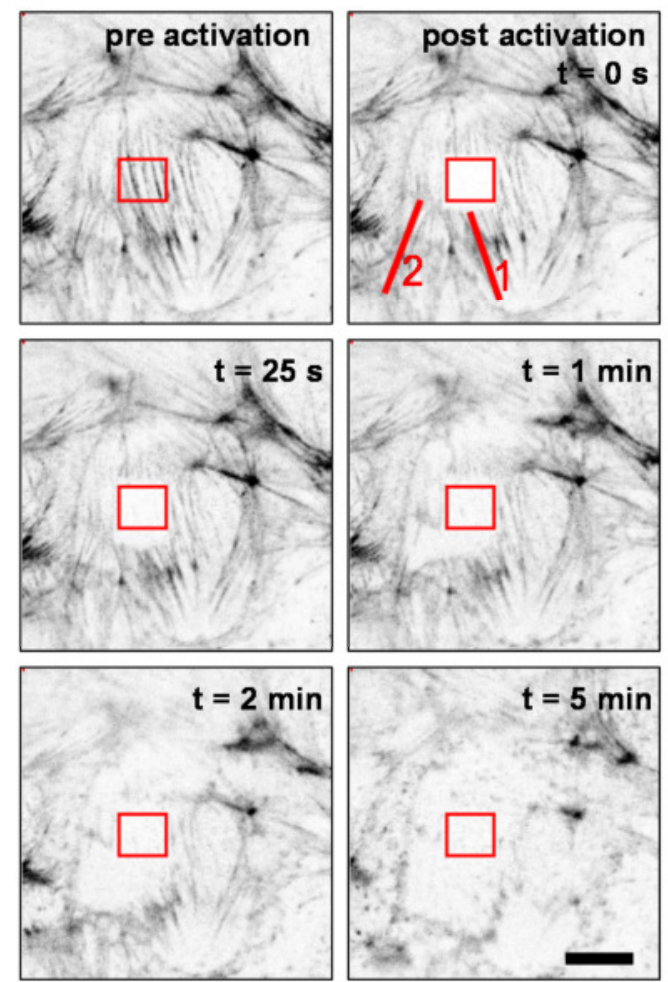

Figure 6. Activation of Nvoc-CytoD within a MDCK cell in a confluent monolayer. MDCK cells were stained with SiR actin. A) Confocal (bottom plane) images of actin fibers taken 2 minutes before illumination (top image) and 7 minutes afterwards (bottom image). The illuminated area is marked with a red square. The yellow dotted frame indicates the region used for the images in B. Arrows highlight actin structures that do not change after illumination. The asterisk denotes structures that are strongly altered. Scale bar: 20 $\mu \mathrm{m}$. B) Images extracted from the yellow square in A at different times. The red frame corresponds to the site of local activation. The red lines shows the regions used to record the kymographs shown in C. Scale bar: $10 \mu \mathrm{m}$. C) Kymographs recorded from the time-lapse over the two lines drawn in $\mathrm{B}$ at $\mathrm{t}=0 \mathrm{~min}$. The calculated velocity for the disappearance of the stress fibers is indicated on the kymograph. 

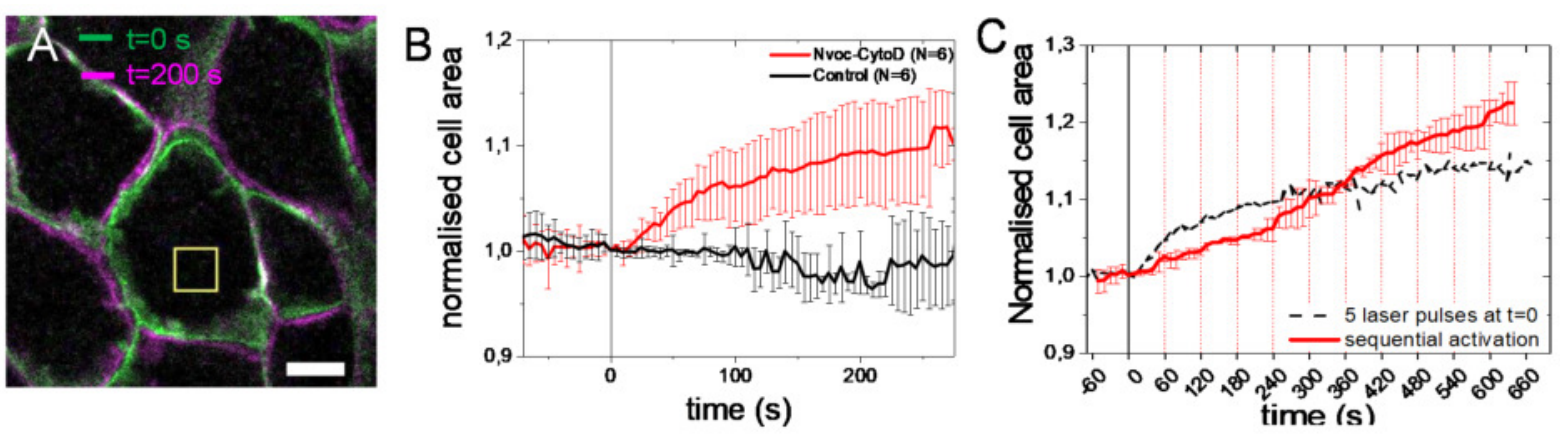

Figure 7. Variation in MDCK cell area upon intracellular activation of Nvoc-CytoD within a confluent monolayer. For illumination a diode laser $(405 \mathrm{~nm})$ was used at a $5 \%$ of is maximal intensity using a 60x Apochromate objective (Zeiss). Dwell time was $131 \mu \mathrm{m}$. A) Exemplary overlayed image of E cadherin staining of a MDCK cell at the time of illumination (green) and $200 \mathrm{~s}$ after the illumination (magenta). Scale bar: $10 \mu \mathrm{m}$. B) Normalized area of illuminated cells measured during 5 minutes after photorelease of CytoD. The exposure dose included 5 consecutive pulses. The red curve show the mean normalised cell area in the presence of $5 \mu \mathrm{M}$ Nvoc-cytoD ( $\mathrm{N}=6$ cells), the black curve represents the control $(0.1 \%$ of DMSO, $\mathrm{N}=6$ ). Error bars represents the standard deviation of the mean. $\mathrm{C}$ ) The red curve shows the normalized area of two cells illuminated with one pulse per minute (noted by the red vertical dotted lines). The black curve is extracted from the data in (B). Between $t=300 \mathrm{~s}$ and $\mathrm{t}=600 \mathrm{~s}$ only 3 cells were measured. 


\section{References}

1. Blanchoin, L.; Boujemaa-Paterski, R.; Sykes, C.; Plastino, J., Actin dynamics, architecture, and mechanics in cell motility. Physiol. Rev. 2014, 94 (1), 235-63.

2. Lukinavičius, G.; Reymond, L.; D'Este, E.; Masharina, A.; Göttfert, F.; Ta, H.; Güther, A.; Fournier, M.; Rizzo, S.; Waldmann, H.; Blaukopf, C.; Sommer, C.; Gerlich, D. W.; Arndt, H.-D.; Hell, S. W.; Johnsson, K., Fluorogenic probes for live-cell imaging of the cytoskeleton. Nature Methods 2014, 11, 731.

3. Hüll, K.; Morstein, J.; Trauner, D., In Vivo Photopharmacology. Chem. Rev. 2018, 118 (21), 10710-10747.

4. $\quad$ Klán, P.; Šolomek, T.; Bochet, C. G.; Blanc, A.; Givens, R.; Rubina, M.; Popik, V.; Kostikov, A.; Wirz, J., Photoremovable Protecting Groups in Chemistry and Biology: Reaction Mechanisms and Efficacy. Chem. Rev. 2013, 113 (1), 119-191.

5. Kowalik, L.; Chen, J. K., Illuminating developmental biology through photochemistry. Nat. Chem. Biol. 2017, 13 (6), 587-598.

6. Suzuki, A. Z.; Sekine, R.; Takeda, S.; Aikawa, R.; Shiraishi, Y.; Hamaguchi, T.; Okuno, H.; Tamamura, H.; Furuta, T., A clickable caging group as a new platform for modular caged compounds with improved photochemical properties. Chem. Commun. 2019, 55 (4), 451-454.

7. $\quad$ Dobber, A.; Phoa, A. F.; Abbassi, R. H.; Stringer, B. W.; Day, B. W.; Johns, T. G.; Abadleh, M.; Peifer, C.; Munoz, L., Development and Biological Evaluation of a Photoactivatable Small Molecule Microtubule-Targeting Agent. ACS Med. Chem. Lett. 2017, 8 (4), 395-400.

8. Gropeanu, R. A.; Baumann, H.; Ritz, S.; Mailänder, V.; Surrey, T.; del Campo, A., Phototriggerable 2' ,7-Caged Paclitaxel. PLoS ONE 2012, 7 (9), e43657.

9. Borowiak, M.; Nahaboo, W.; Reynders, M.; Nekolla, K.; Jalinot, P.; Hasserodt, J.; Rehberg, M.; Delattre, M.; Zahler, S.; Vollmar, A.; Trauner, D.; Thorn-Seshold, O., Photoswitchable Inhibitors of Microtubule Dynamics Optically Control Mitosis and Cell Death. Cell 2015, 162 (2), 403-11.

10. Yahara, I.; Harada, F.; Sekita, S.; Yoshihira, K.; Natori, S., Correlation between effects of 24 different cytochalasins on cellular structures and cellular events and those on actin in vitro. J. Cell Biol. 1982, 92 (1), 69-78.

11. Scherlach, K.; Boettger, D.; Remme, N.; Hertweck, C., The chemistry and biology of cytochalasans. Nat. Prod. Rep. 2010, 27 (6), 869-86.

12. Scherlach, K.; Boettger, D.; Remme, N.; Hertweck, C., The chemistry and biology of cytochalasans. Nat. Prod. Rep. 2010, 27 (6), 869-886.

13. Shoji, K.; Ohashi, K.; Sampei, K.; Oikawa, M.; Mizuno, K., Cytochalasin D acts as an inhibitor of the actin-cofilin interaction. Biochem. Biophys. Res. Commun. 2012, 424 (1), 52-7.

14. Cooper, J. A.; Bryan, J.; Schwab, B., 3rd; Frieden, C.; Loftus, D. J.; Elson, E. L., Microinjection of gelsolin into living cells. J. Cell Biol. 1987, 104 (3), 491-501.

15. Schliwa, M., Action of cytochalasin D on cytoskeletal networks. J. Cell Biol. 1982, 92 (1), 79-91.

16. Godman, G. C.; Miranda, A. F., Cellular contractility and the visible effects of cytochalasin. Front. Biol. 1978, 46, 277-429.

17. Sanger, J. W., The use of cytochalasin B to distinguish myoblasts from fibroblasts in cultures of developing chick striated muscle. Proc. Natl. Acad. Sci. U S A 1974, 71 (9), 36215.

18. Cooper, J. A., Effects of cytochalasin and phalloidin on actin. J. Cell Biol. 1987, 105 (4), 1473-1478.

19. Rampal, A. L.; Pinkofsky, H. B.; Jung, C. Y., Structure of cytochalasins and cytochalasin B binding sites in human erythrocyte membranes. Biochem. 1980, 19 (4), 679-83. 
20. Latorre, E.; Kale, S.; Casares, L.; Gómez-González, M.; Uroz, M.; Valon, L.; Nair, R. V.; Garreta, E.; Montserrat, N.; del Campo, A.; Ladoux, B.; Arroyo, M.; Trepat, X., Active superelasticity in three-dimensional epithelia of controlled shape. Nature 2018, 563 (7730), 203-208.

21. Hetmanski, J. H. R.; de Belly, H.; Busnelli, I.; Waring, T.; Nair, R. V.; Sokleva, V.; Dobre, O.; Cameron, A.; Gauthier, N.; Lamaze, C.; Swift, J.; Del Campo, A.; Starborg, T.; Zech, T.; Goetz, J. G.; Paluch, E. K.; Schwartz, J. M.; Caswell, P. T., Membrane Tension Orchestrates Rear Retraction in Matrix-Directed Cell Migration. Dev Cell 2019, 51 (4), 460475 e10.

22. Nair, U. B.; Joel, P. B.; Wan, Q.; Lowey, S.; Rould, M. A.; Trybus, K. M., Crystal structures of monomeric actin bound to cytochalasin D. Journal of molecular biology 2008, 384 (4), 848-64.

23. del Campo, A.; Boos, D.; Spiess, H. W.; Jonas, U., Surface Modification with Orthogonal Photosensitive Silanes for Sequential Chemical Lithography and Site-Selective Particle Deposition. Angew. Chem. Int. Ed. 2005, 44 (30), 4707-4712.

24. San Miguel, V. n.; Bochet, C. G.; del Campo, A., Wavelength-Selective Caged Surfaces: How Many Functional Levels Are Possible? J. Am. Chem. Sc. 2011, 133 (14), 5380-5388.

25. Lukinavicius, G.; Reymond, L.; D'Este, E.; Masharina, A.; Gottfert, F.; Ta, H.; Guther, A.; Fournier, M.; Rizzo, S.; Waldmann, H.; Blaukopf, C.; Sommer, C.; Gerlich, D. W.; Arndt, H. D.; Hell, S. W.; Johnsson, K., Fluorogenic probes for live-cell imaging of the cytoskeleton. Nat Methods 2014, 11 (7), 731-3.

26. Geiger, B.; Bershadsky, A., Exploring the neighborhood: adhesion-coupled cell mechanosensors. Cell 2002, 110 (2), 139-42.

27. Hutson, M. S.; Tokutake, Y.; Chang, M.-S.; Bloor, J. W.; Venakides, S.; Kiehart, D. P.; Edwards, G. S., Forces for Morphogenesis Investigated with Laser Microsurgery and Quantitative Modeling. Science 2003, 300 (5616), 145.

28. Roy, P.; Rajfur, Z.; Jones, D.; Marriott, G.; Loew, L.; Jacobson, K., Local Photorelease of Caged Thymosin $\beta 4$ in Locomoting Keratocytes Causes Cell Turning. J. Cell Biol. 2001, 153 (5), 1035-1048.

29. Ghosh, M.; Song, X.; Mouneimne, G.; Sidani, M.; Lawrence, D. S.; Condeelis, J. S., Cofilin Promotes Actin Polymerization and Defines the Direction of Cell Motility. Science 2004, 304 (5671), 743.

30. Wu, Y. I.; Frey, D.; Lungu, O. I.; Jaehrig, A.; Schlichting, I.; Kuhlman, B.; Hahn, K. M., A genetically encoded photoactivatable Rac controls the motility of living cells. Nature 2009, 461 (7260), 104-108.

31. Shimizu-Sato, S.; Huq, E.; Tepperman, J. M.; Quail, P. H., A light-switchable gene promoter system. Nat. Biotech. 2002, 20 (10), 1041-1044.

32. Dumbauld, D. W.; Lee, T. T.; Singh, A.; Scrimgeour, J.; Gersbach, C. A.; Zamir, E. A.; Fu, J.; Chen, C. S.; Curtis, J. E.; Craig, S. W.; García, A. J., How vinculin regulates force transmission. Proc. Natl. Acad. Sc. USA 2013, 110 (24), 9788-9793.

33. Riedl, J.; Crevenna, A. H.; Kessenbrock, K.; Yu, J. H.; Neukirchen, D.; Bista, M.; Bradke, F.; Jenne, D.; Holak, T. A.; Werb, Z.; Sixt, M.; Wedlich-Soldner, R., Lifeact: a versatile marker to visualize F-actin. Nat. Methods 2008, 5 (7), 605-7.

34. Schindelin, J.; Arganda-Carreras, I.; Frise, E.; Kaynig, V.; Longair, M.; Pietzsch, T.; Preibisch, S.; Rueden, C.; Saalfeld, S.; Schmid, B.; Tinevez, J.-Y.; White, D. J.; Hartenstein, V.; Eliceiri, K.; Tomancak, P.; Cardona, A., Fiji: an open-source platform for biological-image analysis. Nat. Methods 2012, 9 (7), 676-682. 